(2) Open Access Full Text Article

METHODOLOGY

\title{
Measuring phacoemulsification groove depth using probe tip dimensions and biometry lens thickness readings
}

This article was published in the following Dove Press journal:

Clinical Ophthalmology

17 August 2016

Number of times this article has been viewed

\author{
Kevin Dunne \\ Alex J Buller \\ Department of Ophthalmology, \\ Hawkes Bay District Health Board, \\ Hastings, New Zealand
}

Correspondence: Kevin Dunne

Department of Ophthalmology, Hawkes Bay District Health Board, Omahu Road, Hastings 4I 20, New Zealand

Tel +646878 8I09

Email kevindunne86@gmail.com
Aim: To describe a useful technique utilizing lens thickness from biometric data as well as phacoemulsification (phaco) probe tip dimensions, in order to more accurately determine safe groove depth during divide and conquer techniques for cataract surgery.

Methods: Single center, selection of patients for cataract surgery deemed low risk and suitable for surgical teaching cases. Individual lens thickness measurements from biometry were calculated with known phaco tip dimensions to give an individualized safe number of phaco tip depths for grooving during divide and conquer. This technique was then applied during cataract surgery.

Results: Utilization of this technique allows calculation and determination of an appropriate number of phaco tip depths of grooving for each individual patient. This technique was applied as a teaching tool for surgical trainees, with subsequent successful safe cataract surgeries completed. No posterior capsule ruptures were noted for these cases.

Conclusion: By combining the biometric measurements of an individual patient's lens thickness together with known phaco tip dimensions, individualized safe groove depths can be theoretically determined and applied during divide and conquer cataract surgery.

Keywords: cataract, divide and conquer, groove depth, lens thickness, probe tip

\section{Introduction}

The divide and conquer technique first described by Gimbel ${ }^{1,2}$ in the late $1980 \mathrm{~s}$, has been gradually refined and tested over the years to be safe and reliable. ${ }^{3}$ It involves formation of deep grooves within the lens to allow for fracturing into smaller segments within the capsular bag using the phacoemulsification (phaco) tip and a secondary instrument. This technique is commonly taught first to surgical trainees, due to its advantages of requiring less bimanual manipulation, greater anterior chamber stability, and high reliance on visual cues, ${ }^{4}$ compared to more difficult techniques such as the chop technique. One of the challenging steps of this technique is determining adequate groove depth during sculpting to produce a thin enough posterior plate for effective splitting of the nucleus. Too shallow a groove and the nucleus fails to split, disrupting groove wall integrity, and making further attempts more difficult. Too deep a groove and a posterior capsule rupture is potentially created.

Current methods of groove depth assessment are several, and include utilizing phaco tip diameters. The literature differs in the estimates of how many phaco tips should fit vertically inside a groove, ranging from $2-4 .{ }^{4-6}$ Other visual cues include observing the red reflex, with brightening red reflex suggesting thinning posterior plate; ${ }^{4-6}$ as well as observing for groove floor linear striations parallel to trench long axis, meeting at 
posterior Y-suture. ${ }^{7}$ Visual techniques include utilizing good stereopsis, with better depth perception at lower magnifications. ${ }^{5}$ The parallax technique has also been described, which involves observation of the posterior opacities relative to base of groove when small side to side nuclear displacements are made. Less movement would indicate a deeper groove. ${ }^{5,8}$ This is useful in posterior cortical or subcapsular cataracts where red reflex may be of limited use.

Use of phaco tip diameters is a recognized technique to physically estimate depth however, variables such as phaco probe type and exact lens thickness dimensions can help determine more accurately how many phaco tips depths is the optimum for a particular case.

\section{Patients}

Patients consisted of those undergoing routine cataract surgery and were selected based on appropriateness for trainee operators. Factors considered included ability to remain immobile, minimal preexisting ocular disease, clear corneas, deep anterior chambers, lens stability, and medium density nuclear cataracts.

This report meets the principles set in the Declaration of Helsinki. Written informed consent was obtained from all patients undergoing cataract operations, including material used for teaching and publication purposes, in our hospital (Hawkes Bay Hospital, Hastings, NZ). Given the intraoperative phaco probe depth technique described is commonly already in routine practice, our paper focuses on adding theoretical calculations to allow for the method to be utilized more effectively and safely. As such, this methodology was deemed low risk and out of scope with the NZ Health and Disabilities Ethics Committee, with no application for ethics approval made.

\section{Methods}

In the preoperative stage, appropriate patients are determined for trainee operators. As part of routine preoperative cataract assessment, biometry is done and measurements of lens thickness are often available. With the modern intraocular lens formulas and biometry devices, lens thickness measurements are becoming more commonplace in ophthalmic practice, and are likely to be incorporated into future biometric preoperative measurements routinely.

Knowledge of phaco probe dimensions is crucial for determining probe tip vertical height and official manufacturer product dimensions is ideal. We routinely use a $0.9 \mathrm{~mm}$ Kelman $45^{\circ}$ Mini-Flared ABS tip (Alcon Laboratories, Inc., Fort Worth, TX, USA). The described $0.9 \mathrm{~mm}$ is the internal diameter of the tip aperture whilst the outside diameter

$$
\cos (\theta)=\frac{\text { opp }}{\text { hyp }} \quad \text { hyp }=\frac{\text { opp }}{\cos (\theta)}=\frac{0.85}{\cos (45)} \approx 1.2 \mathrm{~mm}
$$
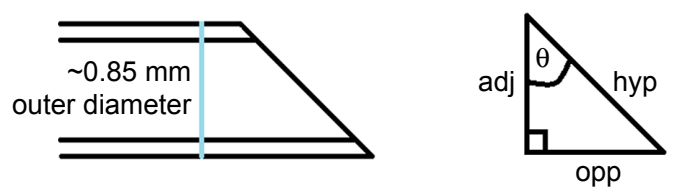

Figure I Calculating phacoemulsification probe aperture diameter using trigonometry.

is actually approximately $1.2 \mathrm{~mm}$, which will also be the vertical height of the phaco tip aperture. Otherwise, one can physically measure the dimensions of the instrument. For those mathematically inclined, one could calculate the aperture diameter for any angled tip, (assuming the probe body diameter was known and the tip was not flared) via the trigonometric equation to calculate the hypotenuse of an angle as shown in Figure 1.

During preoperative planning, an estimate of the required number of phase tip depths for each case can be made, aiming to produce a groove of sufficient depth to enable cracking but maintaining a safe distance from the posterior capsule. For example, if a patient's biometry with a lens thickness of $4.47 \mathrm{~mm}$, using a phaco tip of height of $1.2 \mathrm{~mm}$, an estimated three and half phaco tips would provide adequate depth and still be a safe distance away from the posterior capsule. Keeping this figure in mind, the initial sculpting phase also requires careful recognition of how much of the anterior cortex is removed or disrupted, as measurement will be referenced to the original anterior cortex height. As the grooves are deepened, measurement of depth is done by placing the phaco tip at the deepest central part of the groove in a fashion so that the aperture is vertically oriented as shown in Figure 2, thus achieving expected vertical height of the probe. The top of the probe tip is then measured against the groove wall, and the probe is lifted to place the bottom of the tip

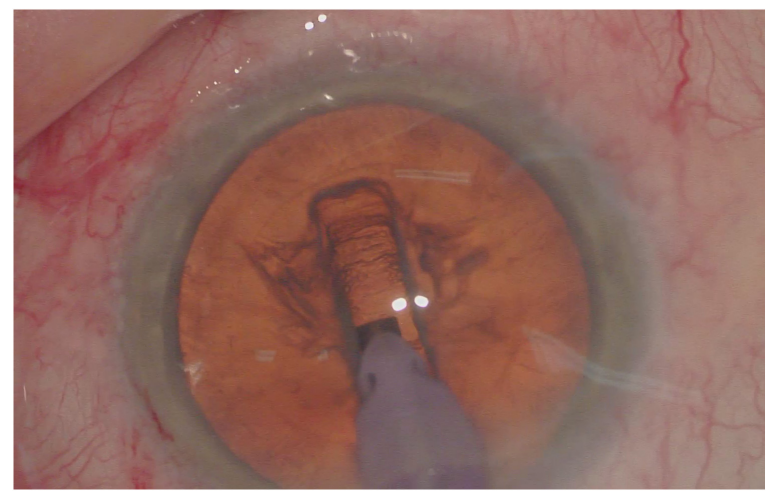

Figure 2 Placing phacoemulsification probe centrally in groove to assess vertical groove height. 
at the previously noted wall height position. This continues until the probe reaches the height of the original anterior cortex and from here further grooving can be decided if the required number of probe tip depths have not been reached. Once the required depth has been reached, splitting of the nucleus proceeds as normal.

\section{Results}

The main results of this described technique allow the operator to calculate the exact number of appropriate probe depths during grooving for any individual patient, as long as preoperative biometry and phaco tip dimensions are known. At the time of writing, this technique has been utilized successfully in a small handful of patients during trainee operated surgery. No posterior capsule rupture occurred during these operations.

\section{Discussion}

With the current available methods of estimating phaco groove depth, many are utilized more effectively once a surgeon gains experience and familiarity with intraoperative findings. To a trainee surgeon however, the knowledge of just how bright a red reflex needs to be before adequate depth is reached is perhaps more difficult to obtain. This can be further complicated by cases where posterior cortical or subcapsular cataract can limit use of red reflexes. Our technique is independent of preexisting lens characteristics or intraoperative findings which may or may not occur in different cases, so can be applied in all cases. This technique can also be helpful when teaching cataract surgery using a microscope that has a monocular view through the side arm viewing eyepiece.

The use of phaco tips as a measurement device provides a physical way to gauge groove depths and can be utilized by both established and trainee cataract surgeons. Previous texts have recommended grooves of anywhere between 2 and 4 phaco tip depths, ${ }^{4-6}$ giving potential groove depths of approximately $2 \mathrm{~mm}$ to $4 \mathrm{~mm}$ but knowing that cataract lens thickness will vary between individuals (one study showing thickness variations of $4.26 \pm 0.55 \mathrm{~mm}),{ }^{9}$ these current recommendations may not be ideal.

By knowing preoperatively the physical dimensions of the phaco probe tip, together with lens thickness readings obtained from biometry, our technique can more accurately predetermine an individualized safe groove depth for any patient undergoing cataract surgery. The ideal thickness for successful lens cracking relies not only on groove depth, but also on other factors such as lens physical characteristics like nuclear density. This technique should allow those that employ it in phacoemulsification to sculpt to a safe groove depth in order to optimize nucleus splitting and minimize potential for posterior capsule breach.

For the trainee cataract surgeon, it has been demonstrated that early in their training, the rates of PC rupture are higher compared to cases performed when more experience has been gained. ${ }^{10}$ This technique should prove especially valuable for early trainees, where knowledge of real time grooving depth can be compared to the desired safe groove depth, allowing for increased confidence, efficiency, and procedural safety awareness.

\section{Conclusion}

By combining the biometric measurements of an individual patient's lens thickness together with known phaco tip dimensions, individualized safe groove depths can be theoretically determined and applied during divide and conquer cataract surgery.

\section{Disclosure}

The authors report no conflicts of interest in this work.

\section{References}

1. Gimbel HV. Divide and conquer (video). Presented at: the European Intraocular Implant Lens Council meeting; 1987.

2. Gimbel HV. Divide and conquer nucleofractis phacoemulsification: development and variations. J Cataract Refract Surg. 1991;17: 281-291.

3. Storr-Paulsen A, Norregaard JC, Ahmed S, Storr-Paulsen T, Pedersen TH. Endothelial cell damage after cataract surgery: divideand-conquer versus phaco-chop technique. J Cataract Refract Surg. 2008;34(6):996-1000.

4. Colvard M, Chang DF, Packer M, Fine H, Hoffman RS. Achieving Excellence in Cataract Surgery a Step-by-Step Approach. Self Published; 2009.

5. Buratto L, Apple DJ, Werner L, Zanini M. Phacoemulsification: principles and techniques. Thorofare, NJ: SLACK Incorporated; 2003.

6. Mahatme V. Step by Step Phacoemulsification: Tips and Tricks. London, UK: CRC press, part of Taylor \& Francis Group; 2005.

7. Kummelil MK, Bhagali R, Das D, Nagappa S, Shetty R, Shetty BK. Systematic transition to phaco-chop. J Vision Sciences. 2015;1(1):28-32.

8. Garg A, Alio JL. Surgical Techniques in Ophthalmology: Cataract Surgery. New Delhi, India: Jaypee Brothers Publishers; 2010.

9. Praveen MR, Vasavada AR, Shah SK, et al. Lens thickness of Indian eyes: impact of isolated lens opacity, age, axial length, and influence on anterior chamber depth. Eye. 2009;23:1542-1548.

10. Corey RP, Olson RJ. Surgical outcomes of cataract extractions performed by residents using phacoemulsification. $J$ Cataract Refract Surg. 1998;24(1):66-72. 


\section{Publish your work in this journal}

Clinical Ophthalmology is an international, peer-reviewed journal covering all subspecialties within ophthalmology. Key topics include: Optometry; Visual science; Pharmacology and drug therapy in eye diseases; Basic Sciences; Primary and Secondary eye care; Patient Safety and Quality of Care Improvements. This journal is indexed on

Submit your manuscript here: http://www.dovepress.com/clinical-ophthalmology-journal
PubMed Central and CAS, and is the official journal of The Society of Clinical Ophthalmology (SCO). The manuscript management system is completely online and includes a very quick and fair peer-review system, which is all easy to use. Visit http://www.dovepress.com/ testimonials.php to read real quotes from published authors. 